\title{
Hydro-mechanical coupling in zero-thickness interface elements, formulation and applications in geomechanics
}

\author{
D. Garolera, I. Aliguer \& I.Carol \\ Dept. of Geotechnical Engineering and Geo-Sciences. Universitat Politècnica de Catalunya. BarcelonaTech \\ (UPC). Barcelona, Spain.
}

\author{
J.M. Segura, M.R. Lakshmikantha \& J. Alvarellos \\ Repsol. Repsol Technology Centre. Móstoles, Spain.
}

\begin{abstract}
Zero-thickness joint/interface elements of the Goodman type, have been advantageously used to solve many problems in solid mechanics involving material interfaces or discontinuities. Some years ago, the authors have also proposed a version of such element for flow/diffusion and hydro-mechanical (H-M) coupled problems, either geomechanical or multiphysics. Some advantages are for instance that fluid pressure discontinuities and localized flow lines may be represented on the same FE mesh used for the mechanical problem, as well as the influence of fluid pressure on mechanical stresses or, conversely, of crack openings on the flow redistribution ("cubic law"). In the paper, previous developments are briefly described, together with some new Geomechanical applications under development, particularly the application to the hydraulic fracture problems, which in the past have been studied mainly via analytical or semi-analytical formulations, or using mixed FE-FD approaches.
\end{abstract}

\section{INTRODUCTION}

Interface or joint elements of zero-thickness type (Goodman et al. 1968), have been successfully used to solve many problems in solid mechanics involving material interfaces or discontinuities. These elements are inserted in between standard elements to allow jumps in the solution field. Their kinematic constitutive ("strain-type") variables are relative displacements, and the corresponding static ("stresstype") variables are stress tractions.

In particular, the authors have used them for representing rock joints in the context of rock masses, contacts between soil and steel reinforcement in reinforced earth structures, or cracks in concrete or other quasi-brittle materials, etc. (Gens et al. 1995, Caballero et al. 2007). Each application requires different constitutive laws, either frictional-type (Gens et al. 1990) or fracture-based with elastoplastic structure (Carol et al. 1997).

Some years ago, the authors have also proposed a version of such element for flow/diffusion, either of geo-mechanical (Segura \& Carol 2004) or multiphysics type (Idiart et al. 2011). Some advantages are for instance that fluid pressure discontinuities and localized flow lines may be represented on the same FE mesh used for the mechanical problem, as well as the influence of fluid pressure on mechanical stresses or, conversely, of crack openings on the flow redistribution (“cubic law”).
More recent developments include advanced “monolithic” implementation (Segura \& Carol 2004), return map algorithms and consistent tangent operator for the constitutive laws and other advanced strategies (Caballero et al. 2008).

Numerical modeling of Hydraulic Fracture (HF), on the other hand, poses some challenges due to discontinuous nature of fracture, and to the strong coupling between the equation that governs the movement (momentum balance) and the equation that controls the fluid pressure (fluid mass continuity). The coupling is due to the mutual influence between fluid and solid: on one side the fluid pressure causes solid deformations including fracture opening, and on the other the fracture opening has dramatic influence on the longitudinal transmissivity along the fracture.

In this paper, hydraulic fracture is reproduced with zero-thickness interface elements along the line started by Segura (2007). In that preliminary study, a fully coupled hydro-mechanic formulation with interfaces was used to simulate a 2D fracture, although the interface behavior was assumed as linear elastic with low moduli. The overall results were in agreement with existing formulas and numerical predictions, although the details of pressure fields showed some unrealistic values to compensate for the constitutive simplifications. In the current approach, zero-thickness interface elements are equipped with non-linear material laws, which leads to a more realist representation of all fields involved including fluid pressure. 


\section{ZERO-THICKNESS INTERFACE ELEMENTS, CONSTITUTIVE LAWS}

Zero-thickness joint or interface elements are finite elements introduced between adjacent continuum elements, with the special feature that they have one less dimension than the standard continuum elements, that is, they are lines in 2D, or surfaces in 3D. The integration of these elements is done through a local orthogonal coordinate system defined on the interface line or surface.

The interface constitutive behavior is formulated in terms of the jump of the main variable across the mid-plane of the interface, and the corresponding force-type conjugate variable. In the standard mechanical problem, those variables are the normal and tangential components of the relative displacements, and their counterpart stress tractions (Fig. 1a).

\subsection{Elastoplastic constitutive law}

The standard interface constitutive model implemented in the code for rock mechanics purposes is a relatively general elastoplastic formulation, which is formulated in terms of normal and shear stress, and the corresponding normal and tangential relative displacements, and includes a hyperbolic failure surface, a range of hardening-softening and dilatancy laws, step-by-step numerical integration, etc. (Gens et al. 1995). However, a simplified version exists that, at the expense of some restrictive assumptions, becomes adequate for computationally efficient explicit integration (Gens et al. 1995). The main simplifying assumptions are perfect plasticity, no dilatancy, and a linear elastic relationship between the normal stress and the normal relative displacement in compression (zero normal stress in tension).

The yield surface in the $\sigma-\tau$ plane, where $\tau=$ $\sqrt{\tau_{1}^{2}+\tau_{2}^{2}}$ is defined by:

$$
F=\tau^{2}-\tan ^{2} \phi\left(\sigma^{2}-2 a \sigma\right)=0
$$

Due to the expression of the yield surface and the elastic relationship between the normal stress and the normal relative displacement, once the normal stress is known, the ratio between $\tau_{1}$ and $\tau_{2}$ is the only unknown in the integration of the constitutive law. The angle $\lambda$, which represents this ratio, can be obtained using the following equation:

$$
\tan \left(\frac{\beta-\lambda}{2}\right)=\tan \left(\frac{\beta-\lambda_{0}}{2}\right)\left(\frac{\tau+\sqrt{\tau^{2}+a^{2} \tan ^{2} \phi}}{\tau_{0}+\sqrt{\tau_{0}^{2}+a^{2} \tan ^{2} \phi}}\right)^{-\frac{K_{t} \Delta v}{K_{n} \Delta u \tan \phi}}
$$

Where $\beta$ relates the imposed tangential relative displacements $\Delta v_{1}$ and $\Delta v_{2}, K_{n}$ and $K_{t}$ are the normal and shear stiffness moduli respectively and $\lambda_{0}$ is the previous ratio between $\tau_{1}$ and $\tau_{2}$. More details of this simplified model can be found in (Gens et al. 1995)
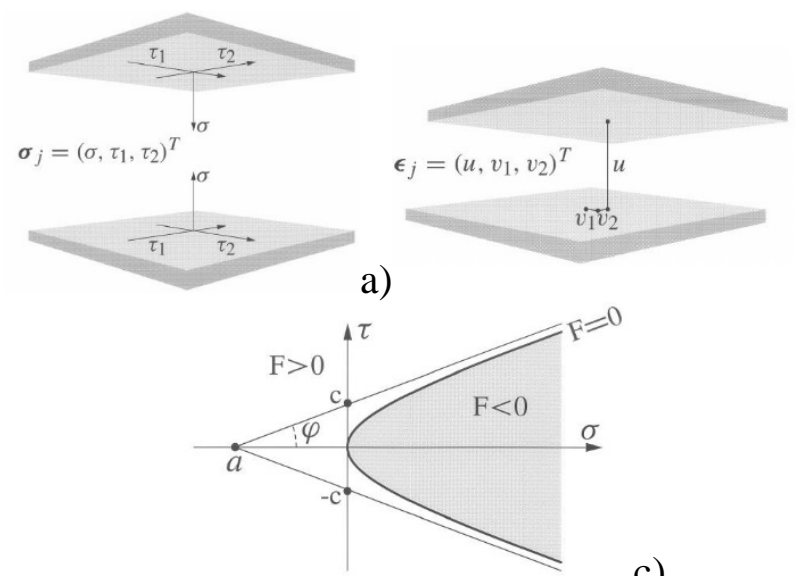

b)

c)

Figure 1. Constitutive model stress and displacement variables definition (a, b). Yield surface definition (c).

\subsection{Hydro-mechanical formulation for interface elements}

Darcy flow is assumed in the continuum elements, and a conceptually similar approach including longitudinal and transversal flow is used for the interface elements [5a]. The coupling is formulated via the influence of deformation on permeability and water content of both continuum and interfaces, in this case using a monolithic coupled approach, i.e. solving simultaneously the momentum balance (eq. 3) and the fluid mass continuity (eq. 4):

$$
\begin{gathered}
\int_{\Omega}-\boldsymbol{B}^{T} \boldsymbol{\sigma}_{n+\theta} d \Omega+\boldsymbol{Q} \overline{\boldsymbol{p}}_{n+\theta}+\boldsymbol{f}_{n+\theta}^{u}=\mathbf{0} \\
\Delta t_{n+1} \boldsymbol{H}_{n+\theta} \overline{\boldsymbol{p}}_{\mathrm{n}+\theta}+\boldsymbol{Q}^{T} \Delta \overline{\mathbf{u}}_{n+1}+\boldsymbol{S} \Delta \bar{p}_{n+1}- \\
\Delta t_{n+1} f_{n+\theta}^{p}=0
\end{gathered}
$$

In the equations above, $\boldsymbol{B}$ is the classical straindisplacement matrix, $\boldsymbol{\sigma}$ is the stress tensor, $\boldsymbol{Q}$ is the coupling matrix, $\overline{\boldsymbol{p}}$ is the fluid pressure vector, $t$ is time, $\boldsymbol{H}$ is the diffusion matrix, $\overline{\mathbf{u}}$ is the displacement vector, $\boldsymbol{S}$ is the storage matrix and $\boldsymbol{f}$ is the right-hand term of the equilibrium equations, for more details see Segura \& Carol (2004).

Focusing on the interfaces, the diffusion matrix is composed of two terms (Fig. 2), one related to longitudinal flow and the other to transversal flow. The longitudinal fluid continuity equation is given by:

$$
\frac{\partial Q_{l}}{\partial l}+\frac{1}{M} \frac{\partial p_{m}}{\partial l}+\alpha \frac{\partial A_{n}}{\partial t}=0
$$

where the total discharge is related to the gradient of pressure via a Darcy-like equation

$$
Q_{l}=-T_{l}\left(\frac{1}{\gamma_{f}} \frac{\partial p_{m}}{\partial l}+\frac{\partial z_{m}}{\partial l}\right)
$$

and the longitudinal transmissivity is defined according to the cubic law:

$$
T_{l}=\frac{g}{12 v} A_{n}^{3}
$$

On the other hand, the transversal flow is assumed to depend on the pressure jump across the discontinuity, with transversal conductivity $K_{t}$ 


$$
q_{t}=K_{t}\left(p^{b o t}-p^{t o p}\right)
$$

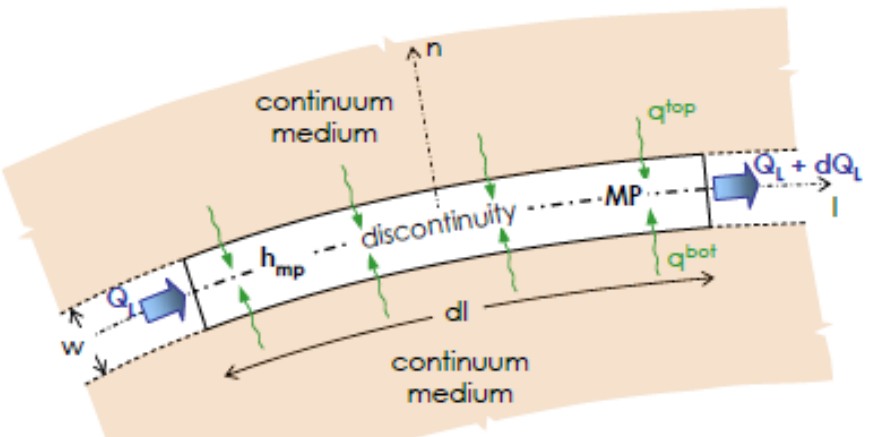

Figure 2. Flow through the differential zero-thickness interface element (Segura 2007).

Assumptions (eqs. 5-8) lead to an element-level coupled system for the interface elements similar to (eqs. 3-4), and therefore can then be treated in the code in the same way as the similar equations for continuum elements (Segura \& Carol 2004, Caballero et al. 2008).

\section{SINGLE 2D HYDRAULIC FRACTURE STUDY}

The above formulation has been implemented in an in-house developed general-purpose FE code, and applied to the study of H-M coupled problems. The first example presented in this paper is the analysis of a single hydraulic fracture in 2D. For the purpose of verification, the geometry and parameters have been taken from the work of Boone \& Ingraffea (1990), who combined 2D Finite Elements for the mechanical behavior, with 1-D Finite Differences for the flow along the crack channel.

A semicircle domain of radius $80 \mathrm{~m}$ representing a transversal cross-section of the borehole and fracture, as depicted in Figure 3a, is discretized with the standard linear triangular finite element mesh of Figure $3 b$. The fracture is inserted along the abscissa axis with double node linear interface elements, as shown in Figure 3c.

The boundary and load conditions are applied in two steps (Figure 4):

- A distributed load of $1.0 \mathrm{MPa}$ is applied over the outer boundary in order to simulate the in-situ initial stress. Initial pore pressure is assumed with constant null value at the boundary and flow analysis for this initial stress calculation is run as steady state, with a resulting zero pressure in the entire domain.

- A fluid is injected at the fracture mouth with constant $\mathrm{Q}=0.0001 \mathrm{~m}^{3} / \mathrm{s}$. The remaining boundary conditions defined in step 1 are maintained. This step is run under transient conditions with increasing time steps until a total duration of 25 seconds.
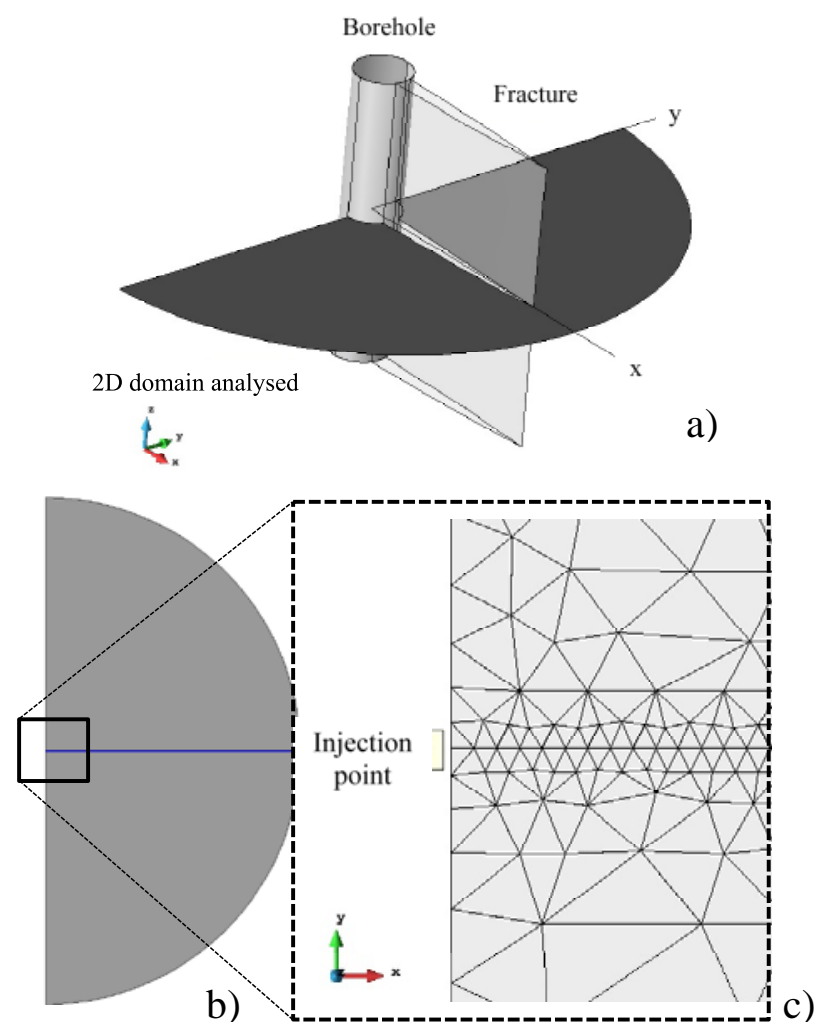

Figure 3. Model description: a) scheme of the hydraulic fracture test; b) FE mesh, and b) detail of discretization at injection point.

The continuum elements are assumed linear elastic and isotropic. With regard to flow, two scenarios are considered, one without leak-off (impervious case), and another one with low leak-off (pervious case). All parameters are displayed in Table 1.

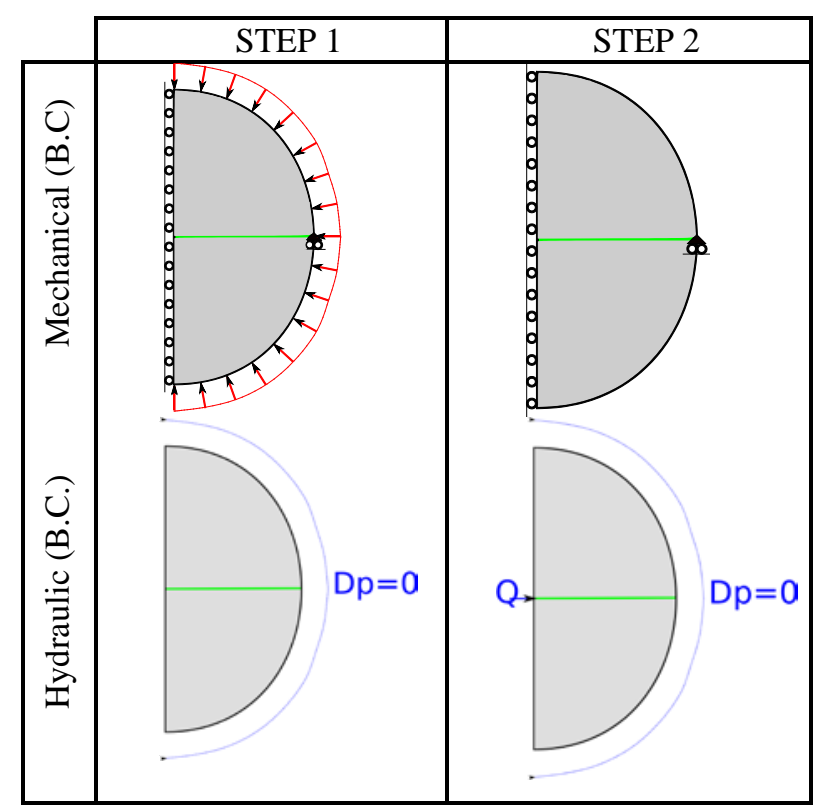

Figure 4. Boundary conditions for mechanical (upper diagrams) and flow (lower diagrams), for each of the two steps of the analysis (vertical columns).

The interface elements are assumed to behave according to the elasto-plastic model described above. Normal and shear elastic stiffness are set to very high values, which can be understood as penalty 
coefficients in order to minimize elastic deformations including interpenetration. As the result, practically all the relative displacement will correspond to plastic deformations representing opening/sliding of the interface. Parameters of the loading surface (assumed fixed in this analysis, perfect plasticity) represent a lower bound scenario of the interface strength, in particular tensile strength is assumed zero as in Boone \& Ingraffea (1990).

The hydraulic behavior of the interface is assumed to follow "cubic law" (eq.7). Table 2 shows the parameters used for this study.

Table 1: Material properties of continuum.

\begin{tabular}{|c|c|c|}
\hline & Impervious & \\
\hline E (Young modulus) & $14,400.0$ & $\mathrm{MPa}$ \\
\hline$v$ (Poisson ratio) & 0.2 & - \\
\hline K (hydraulic conductivity) & $1 \times 10^{-25} \quad 2 \times 10^{-7}$ & $\mathrm{~m} / \mathrm{s}$ \\
\hline $\begin{array}{l}\text { Ks (skeleton } \\
\text { compressibility) }\end{array}$ & 36000.0 & $\mathrm{MPa}$ \\
\hline$\alpha$ Biot & 0.0 & \\
\hline
\end{tabular}

Table 2: Material properties of interfaces.

\begin{tabular}{lll}
\hline Kn (Normal stiffness) & $1,000,000.0$ & $\mathrm{MPa} / \mathrm{m}$ \\
\hline Kt (Tangential stiffness) & $1,000,000.0$ & $\mathrm{MPa} / \mathrm{m}$ \\
\hline $\tan \phi$ (friction angle) & 0.577 & - \\
\hline $\mathrm{a}$ (apex) & 0.0 & $\mathrm{MPa}$ \\
\hline $\mathrm{c}$ (cohesion) & 0.0 & $\mathrm{MPa}$ \\
\hline $\begin{array}{l}\mathrm{T}_{10} \text { (initial longitudinal } \\
\text { transmissivity) }\end{array}$ & 0.0 & $\mathrm{~m}^{2} / \mathrm{s}$ \\
\hline $\begin{array}{l}\mathrm{K}_{\text {trans }} \text { (Transversal } \\
\text { conductivity) }\end{array}$ & 1.0 & $1 / \mathrm{s}$ \\
\hline$\alpha$ Biot & 1 & \\
\hline M Biot & $1 \times 10^{9}$ & \\
\hline
\end{tabular}

\subsection{Impervious case (no leak-off), and comparisons to existing literature.}

The problem stated is sufficiently simple to allow us to obtain a closed-form analytical solution. Spence \& Sharp (1985) and GDK (Geertsma \& De Klerk 1969, Geertsma \& Haaikens 1979) obtain the following expressions for the fracture length, crack mouth opening displacement (CMOD) and pressure at the crack mouth:

$$
\begin{aligned}
& L=A\left(\frac{G Q^{3}}{\mu(1-v)}\right)^{1 / 6} t^{2 / 3} \\
& \text { CMOD }=B\left(\frac{\mu(1-v) Q^{3}}{G}\right)^{1 / 6} t^{1 / 3} \\
& P_{c m}=C\left(\frac{G^{3} Q \mu}{(1-v)^{3} L^{2}}\right)^{1 / 4}+S
\end{aligned}
$$

In those formulae, $A, B$ and $C$ take the values 0.65 , 2.14 and 1.97 for Spence's model, and 0.68, 1.87 and 1.38 for the GDK model.

The results obtained in our calculations, together with those two formulae, and the numerical results by Boone \& Ingraffea (1990) for an injection of 25 seconds, are shown in Figures 5-6. The curves exhibit a relatively good coincidence which seems quite reassuring.

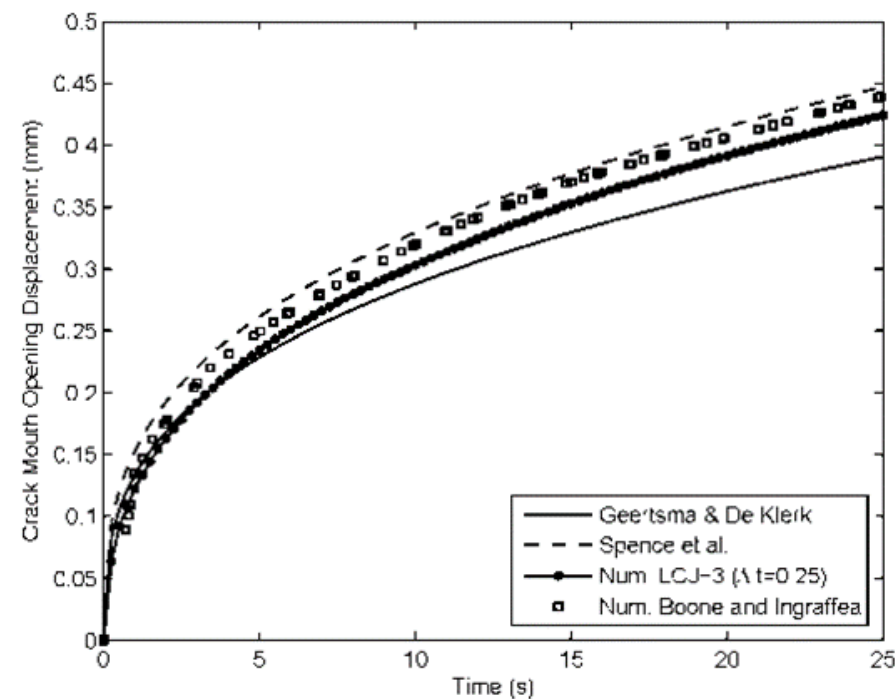

Figure 5. Crack mouth opening displacement (CMOD) evolution with time for a 25 s injection case.

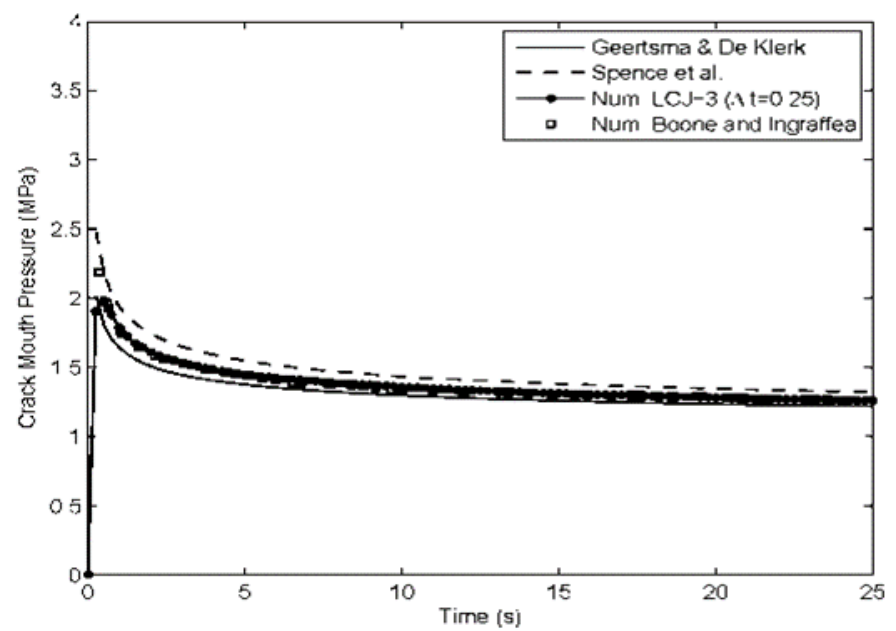

Figure 6. Crack mouth pressure evolution with time for a 25s injection case.

Figure 7 represents the pressure profile along the fracture at the final stage of $25 \mathrm{sec}$, both for the present calculation and for the Boone \& Ingraffea (1990) paper. The match is quite good except near the crack tip, which seems possible given the different approaches for the modeling of flow continuity. Note in this figure, the negative pressure near the crack tip known as "fluid lag", which has been obtained in the proposed model as the result of the delay between opening of the crack and filling with fluid, which in an impermeable medium can only reach the crack tip along the fracture itself.

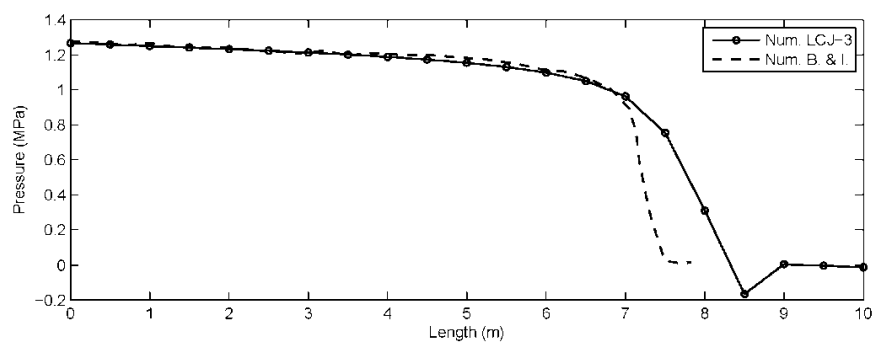

Figure 7. Comparison of pressure profile with results from Boone \& Ingraffea at $25 \mathrm{sec}$, note fluid lag predicted. 


\subsection{Pervious case (with leak-off)}

The only changes with regard to the previous section are the consideration of permeable rock, and the insitu stress, with similar values to the ones used in Boone \& Ingraffea (1990), in particular:

- Continuum permeability $\mathrm{K}=2 \cdot 10^{-7} \mathrm{~m} / \mathrm{s}$.

- Initial in-situ stress 1.2 $\mathrm{MPa}$ (total = effective in this case)

Note that in this case, no analytical formula exists and the only comparison can be done with the results published in that reference. As a novelty, the amount of fluid being leaked into the rock continuum can also be evaluated. The original reference only gives results for the first 10 seconds and so initial comparisons (Figs. 8-9) are made for that time.

\section{CONCLUDING REMARKS}

The modeling approach based on finite elements with zero-thickness interface elements including both solid mechanics, fluid flow and coupling aspects via cubic law, formulated in a monolithic fashion, seems well suited to represent the phenomenon of hydraulic fracturing. In the single fracture case presented, the results obtained exhibit a good match to classical formulae and existing literature, and extends the prediction consistently to longer injection times. Ongoing developments aim at extending the model presented to $3 \mathrm{D}$, multiple interacting fractures and other challenging situations.

More details and additional results can be found in Garolera et al. (2013).

\section{ACKNOWLEDGEMENTS}

The work was partially supported by research grants BIA2012-36898 from MEC (Madrid), which includes FEDER funds, and 2009SGR-180 from Ge-neralitat de Catalunya (Barcelona). The support from REPSOL for this research is also greatfully acknowledged.

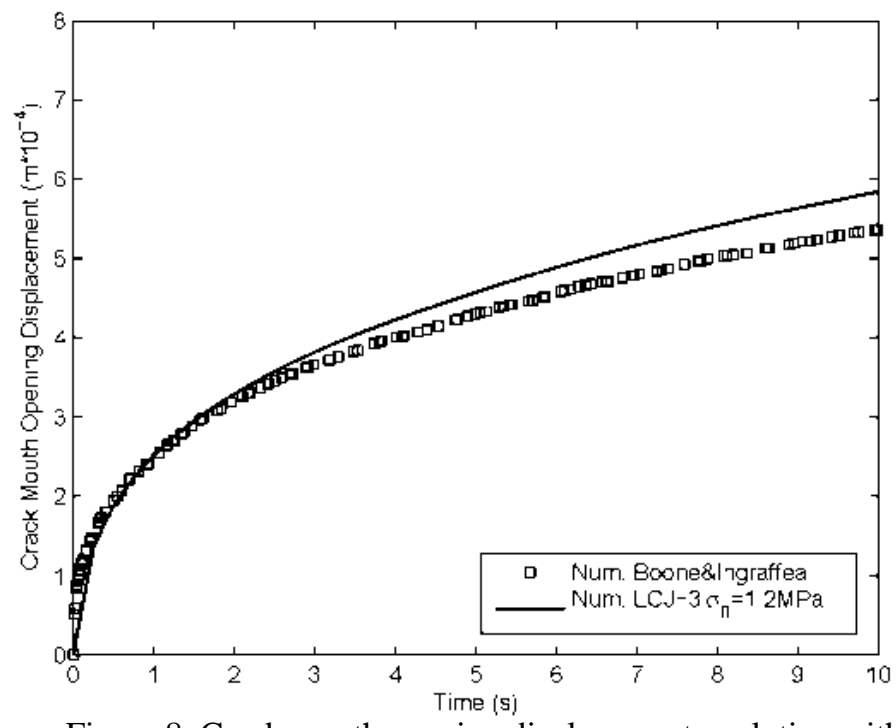

Figure 8. Crack mouth opening displacement evolution with time up to $10 \mathrm{sec}$. Result compared with Boone \& Ingraffea (1990).

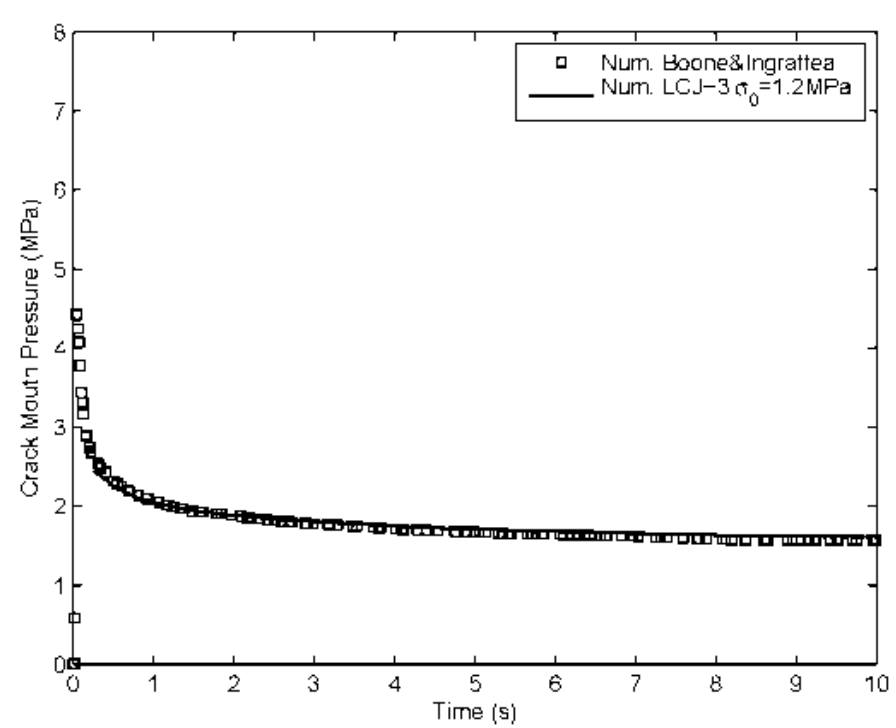

Figure 9. Crack mouth pressure evolution with time up to 10 sec. Result compared with Boone \& Ingraffea (1990).

\section{REFERENCES}

Boone, T.J. \& Ingraffea, A.R. 1990. A numerical procedure for simulation of hydraulically-driven fracture propagation in poroelastic media, Int. J. Numer. Anal. Meth. Geomech., 14: 27-47.

Caballero, A., Carol, I. \& López, C.M. 2007. 3D mesomechanical analysis of concrete specimens under biaxial loading, Fract. and Fatigue of Engrg. Mater, 30: 877886.

Caballero, A., Willam, K. \& Carol, I. 2008. Consistent tangent formulation for 3D interface modeling of cracking/fracture in quasi-brittle materials., Computer Methods In Applied Mechanics And Engineering, 197: 2804-2822.

Carol, I., Prat, P. \& López, C.M. 1997. A normal/shear cracking model. Application to discrete crack analysis, ASCE J. Engrg. Mech., 123(8): 765-773. 
Garolera, D., Aliguer, I., Segura, J.M., Carol, I., Lakshmikantha, M.R. \& Alvarellos, J. 2013. Zero-thickness interface elements with $\mathrm{H}-\mathrm{M}$ coupling, formulation and applications in geomechanics. In XII International Conference on Computational Plasticity. Fundamentals and Applications COMPLAS XII. Oñate, E., Owen, D.R.J., Peric, D., \& Suárez B. (Eds). pp. 1372-1383.

Geertsma, J. \& De Klerk, F. 1969. A rapid method of predicting width and extent of hydraulic induced fractures, J. Pet. Tech., 246: 1571-1581.

Geertsma, J. \& Haaikens, R. 1979. A Comparison of the Theories for Predicting Width and Extent of Vertical Induced Fractures, J. Energy Resources Tech, 101: 8-19.

Gens, A., Carol, I. \& Alonso, E. 1990.A constitutive model for rock joints formulation and numerical implementation, Computers and Geotechnics, 9(1-2): 3-20.

Gens, A., Carol, I. \& Alonso, E. 1995. Rock joints: FEM implementation \& applications. In Mechanics of Geomaterial Interfaces, A. Selvadurai \& M. Boulon, Eds., Elsevier, 395-420.

Goodman R., Taylor, R. \& Brekke, T. 1968. A model for the mechanics of jointed rock, Journal of Soil Mechanics and Foundations Division, 99: 637-659.

Idiart, A., López, C.M. \& Carol, I. 2011. Chemo-mechanical analysis of concrete cracking and degradation due to external sulfate attack: A meso-scale model, Cement \& Concrete Composites, 33: 411-423.

Segura, J.M. \& Carol, I. 2004. On zero-thickness interface elements for diffusion problems, International Journal for Numerical and Analytical Methods in Geomechanics, 28(9): 947-962.

Segura, J.M. 2007. Coupled HM analysis using zero-thickness interface elements with double nodes, $\mathrm{PhD}$ dissertation at Universitat Politècnica de Catalunya. BarcelonaTech (UPC). Spain.

Spence, D. \& Sharp, P. 1985. Self-Similar Solutions for Elastohydrodynamic Cavity Flow, Proc. R. Soc. Lond. A, 400 (1819): 289-313. 